\title{
ANALISE COMBINATÓRIA: UMA APRENDIZAGEM AO CALCULO FINITO DE POSSIBILIDADES DO CUBO DE RUBIKS
}

\author{
Christian Luz Pelissari de Oliveira, Fernando Pereira de Souza. \\ Universidade Federal de Mato Grosso do Sul - UFMS, Grupo PET Conexões de Saberes - Matemática, Três \\ Lagoas, MS. E-mail: christian pelissari@hotmail.com. \\ Agência de fomento: Programa de Educação Tutorial
}

\section{RESUMO}

O presente artigo é resultado de um trabalho de pesquisa do Curso de Licenciatura em Matemática no âmbito do Programa de Educação Tutorial. O objetivo é explorar aspectos desenvolvendo o raciocínio lógico e combinatório relacionado à resolução geométrica do Cubo Mágico, com intuito de analisar seu comportamento prático, assim buscando auxílios para complementar o ensino nas aulas de Matemática, transformando as interessantes para os alunos. O desenvolvimento do trabalho se teve, através de estudo teórico, prático e pesquisa bibliográfica, envolvendo seminários, exercícios e interpretações de análise combinatória. Após o estudo de todas as possíveis soluções combinatórias do Cubo Mágico pode-se notar que com a exposição de práticas desperta no aluno a curiosidade e motivação para o aprendizado de conceitos matemáticos.

Palavras-chave: Cubo Mágico, Permutações, Combinações, Ensino de Matemática, Programa de Educação Tutorial.

\section{COMBINATORIAL ANALYSIS: A LEARNING FINITE CALCULATION CUBE POSSIBILITIES RUBIKS}

\section{ABSTRACT}

The present article is the result of a research work of Degree in Mathematics in the Tutorial Education Program. The aim is to explore ways to develop the logic and combinatorial reasoning related to the geometric resolution of the Rubik's magic cube, in order to analyze their practical behavior, thus seeking aid to supplement teaching in mathematics classes, transforming interesting for students. The development work has had, through theoretical study and practical literature, involving seminars, exercises and combinatorics interpretations. After studying all possible Rubik's magic cube combinatorics solutions, can be noticed that practical exposure arouse student's curiosity and motivation for learning mathematical concepts.

Keywords: Magic Cube, Permutations, Combinations, Teaching Math, Tutorial Education Program. 


\section{INTRODUÇÃO}

O Cubo Mágico, também conhecido como Cubo de Rubik, é um brinquedo da década de 70, um quebra cabeça mecânico tridimensional como o nome mesmo diz, o brinquedo apresenta a forma espacial geométrica de um hexaedro (Cubo). Inventado pelo arquiteto Húngaro Erno Rubik. Também utilizado em suas aulas explicando a geometria espacial, chegando ao ponto de ter de ficar isolado do mundo por cerca de um mês para encontrar uma solução, assim resolvendo seu quebra cabeça. Este engenho mágico tornou-se popular rapidamente na época e nos dias de hoje como o brinquedo mais conhecido da história. O Cubo pode ser um grande instrumento de auxilio no aprendizado aos alunos, contribuindo como um recurso mecânico de interação, despertando a curiosidade do "saber", como a matemática pode ocorrer em um simples objeto, mas de enorme complexidade. Contudo buscando uma vasta participação dos alunos. Ressaltando que todas as imagens foram elaboradas pelo os autores a partir de fotografias feitas com aparelho smartphone, assim, a arte final com auxilio do software de Edição de imagens.

O objetivo do presente trabalho se da em explorar o Cubo Mágico, seus aspectos físicos, mecânicos. Fazendo um estudo analítico de seu corpo, todos seus plausíveis movimentos em sua geometria. Consequentemente, chegando ao método possibilitando o cálculo total de procedimentos que há para a montagem do engenho de Rubiks.

\section{METODOLOGIA}

O trabalho foi desenvolvido através de estudo teórico, prático e pesquisa bibliográfica, que incluiu apresentação de seminários, resolução de exercícios, interpretações analiticamente combinatória e de movimentos na geometria espacial do cubo.

\section{RESULTADOS}

\section{CONHECENDO O QUEBRA CABEÇA}

O Cubo de Rubiks contém em sua forma, seis faces, ambas com cores distintas, amarelo, verde, vermelho, azul, laranja e branca. Sua estrutura disposta em ( $3 \times 3 \times 3$ ) por 54 quadradinhos, ligados em 26 peças em sua armação central, desta possibilitando seus movimentos rotacionais, como mostrado na figura 1:

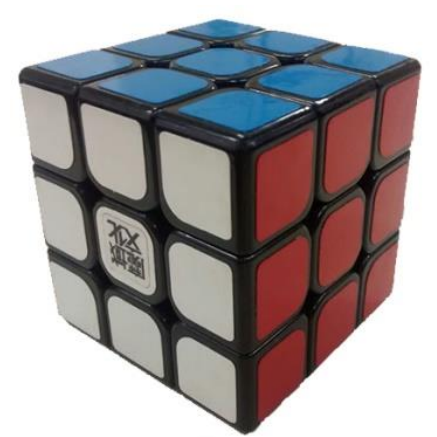

Figura 1 - Cubo Mágico

Deste modo seu esqueleto é dividido em três peças específicas: centro, meio e cantos. Existe uma única peça central em todas as faces, na qual, contendo seis peças desta em todo cubo, apresentando apenas uma cor. Logo, claramente essa peça central determinará a cor específica de cada face. Como na figura 2: 


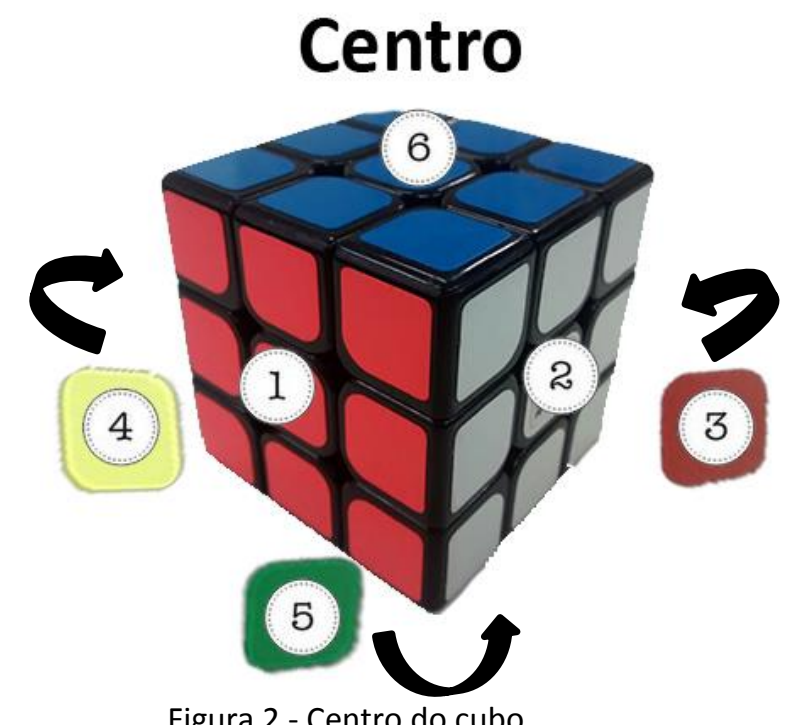

Figura 2 - Centro do cubo

As peças denominadas como "meio", são peças constituídas por duas cores diferentes, que ficam no meio de cada aresta. Em seu corpo existem doze peças de duas cores como mostra a figura 3.

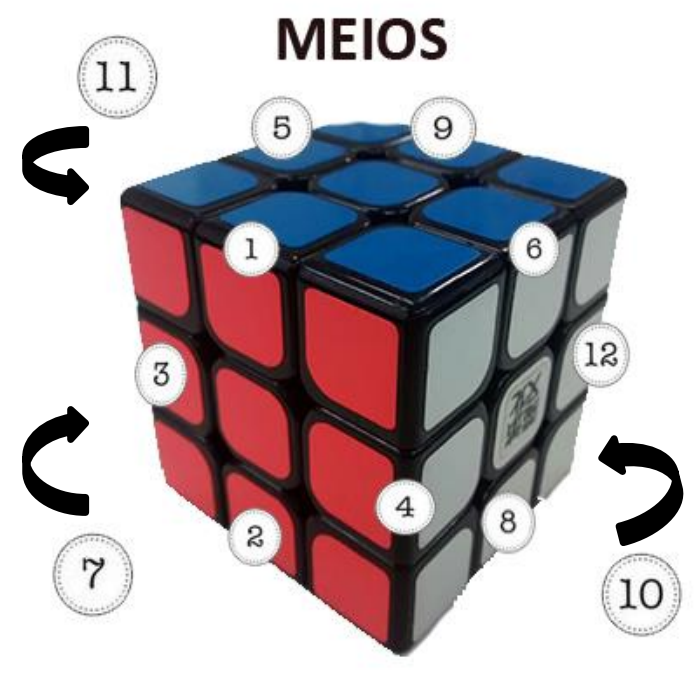

Figura 3 - Meios do Cubo Mágico

As "quinas ou cantos", são as que contêm três cores, ficam localizadas no vértice do cubo, tendo um total de oito peças, veja a figura 4. 


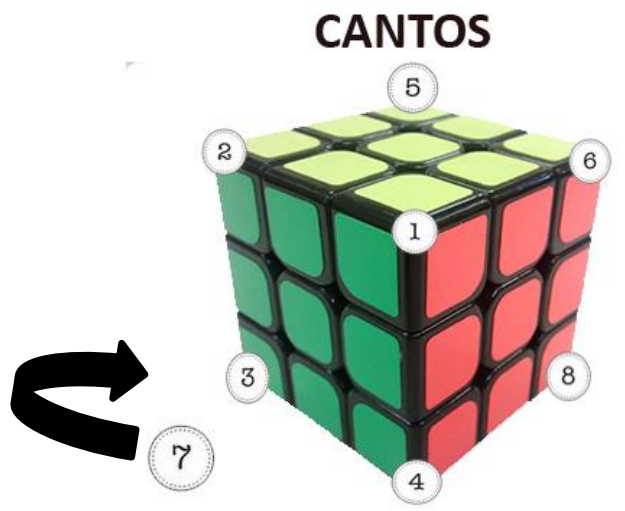

Figura 4 - Cantos do Cubo Mágico

A face do cubo contém um conjunto de nove quadrados menores, que se movimentam para ambos os sentidos. A cada movimento, automaticamente, estará movimentando quatro meios, quatro quinas e rotacionando o centro.

\section{FACE}

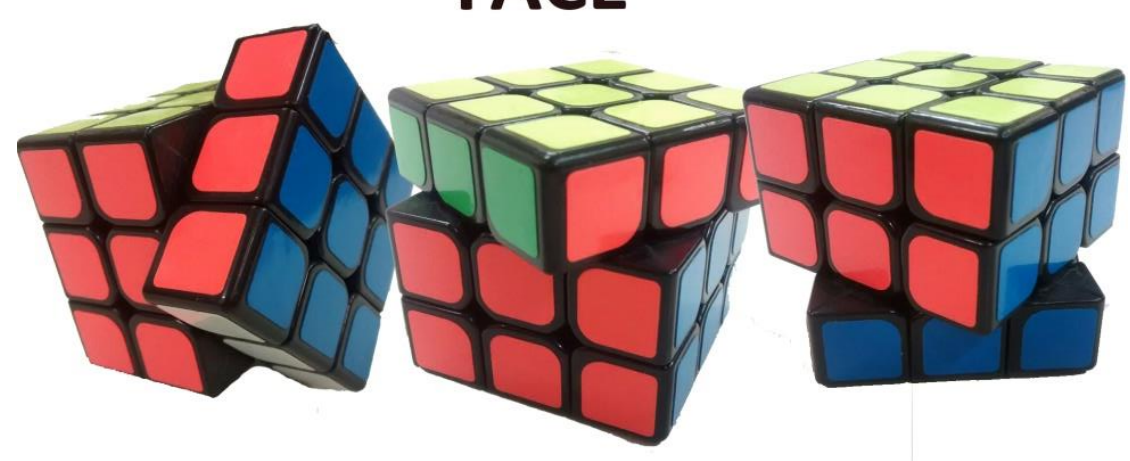

Figura 5 - Movimento das faces

O objetivo do Cubo se ocorre após embaralhamento arbitrário chegando a um aspecto aleatório, assim, deste modo, movimentando os lados, remodelando o quebra cabeça até voltar em sua forma, com todas as faces tenham a mesma cor, como mostra a figura 6 :
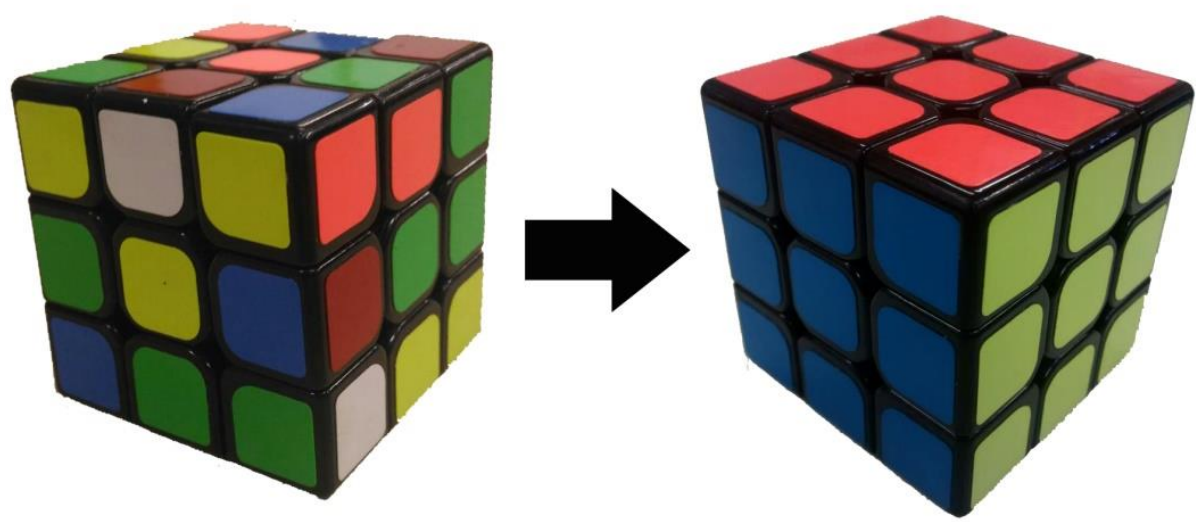

Figura 6 - Montagem do cubo 
Desta tiramos uma primeira observação perante as respectivas peças e seus movimentos. A cada movimento executado, sempre a "central", continuará no centro do cubo, fazendo apenas movimentos de rotação no seu próprio eixo. A do "meio" se permutara em todo corpo do cubo, sendo que necessariamente estará no meio de cada aresta do mesmo. Assim, os "cantos" também permutarão em todo o cubo, mas com a mesma restrição, que a cada movimento, mudará de posição para outro canto.

\section{COMBINAÇÕES POSSÍVEIS}

A fim de estimarmos o número de possibilidades existentes para o Cubo Mágico $(3 \times 3 \times 3)$, devemos aplicar a Análise Combinatória. Vamos supor que queremos descobrir todas as possíveis combinações com três letras $A, B$ e $C$ sem que haja nenhuma repetição, ou seja, teremos seis possibilidades $(A B C, A C B, B A C, B C A, C A B$ e $C B A)$, com isso ao invés de contar todas as possibilidades efetua-se o calculo $3 \cdot 2 \cdot 1=6$, pois temos três possibilidades para a escolha da primeira letra, depois duas e por fim uma, logo podendo usar a notação 3 !

Deste modo, primeiramente analisaremos os vértices, logo temos oito quinas que podem permutar e ser ocupadas por qualquer uma delas. Desta forma calculamos o número de permutações possíveis, somente dos vértices será de 8!. Chegando ao número de 40.320 permutações.

Temos os meios das arestas, sendo 12 meios que permutam também em todo o quebra cabeça. Calculando todas as combinações, assim teremos 12! Ou seja, 479.001 .600 permutações.

Podemos pensar então que as possibilidades de montar um cubo mágico é de 8 ! · 12 !. No entanto, o cubo mágico possui algumas particularidades. Com isso, estudando os cantos com uma maior profundidade, percebemos que os cantos também podem ser rotacionados, possuindo três cores que podem ser giradas em cada canto sozinhas, poderíamos pensar em $3^{8}$, mas isso seria um absurdo, pois estamos alegando um caso impossível, veja a figura 7:

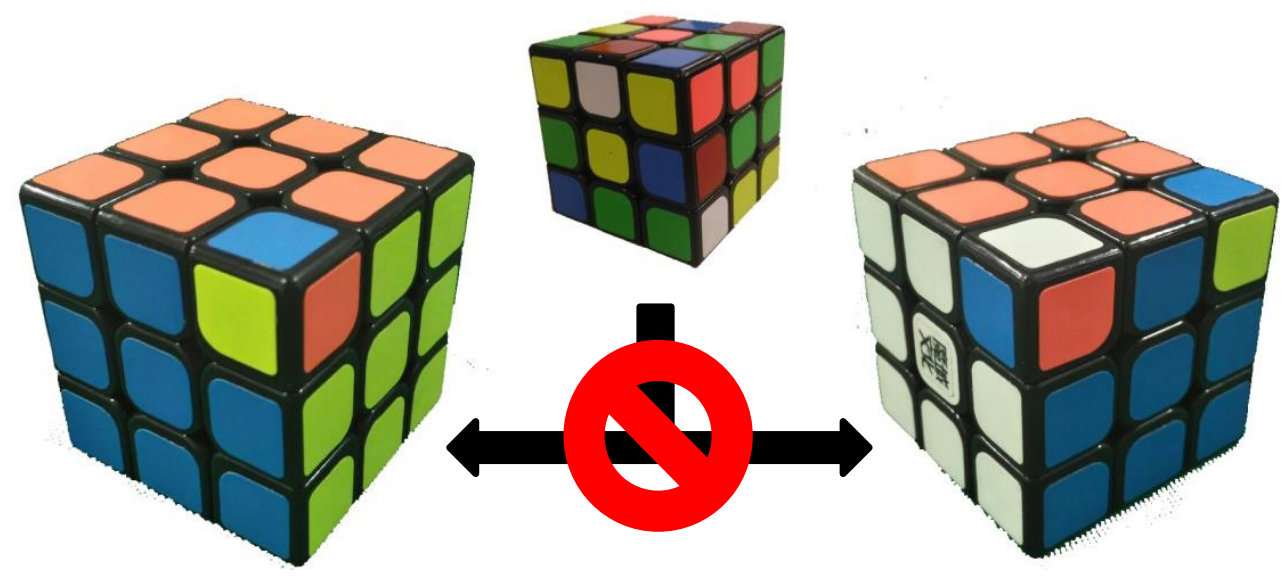

Figura 7 - Caso impossível dos cantos

Os cantos são orientados por existir uma conexão entre eles, os cantos devem ser girados de três em três para o mesmo sentido ou podem ser dois cantos sendo um no sentido horário e outro no sentido anti-horário. Logo, não é possível movimentar apenas dois cantos no mesmo sentido. Com isso, apenas $\left(\frac{1}{3}\right)$ das $3^{8}$ são possíveis, sendo $\left(\frac{3^{8}}{3}\right)$, assim resultando a $3^{7}$, logo temos 2187, permutações rotacionadas. Contudo o mesmo raciocínio deve ser realizado para os meios, poderíamos pensar $2^{12}$, chegamos à mesma contradição, pois os meios são transportados aos pares, não sendo possível orientar um nem três meios sozinhos. 

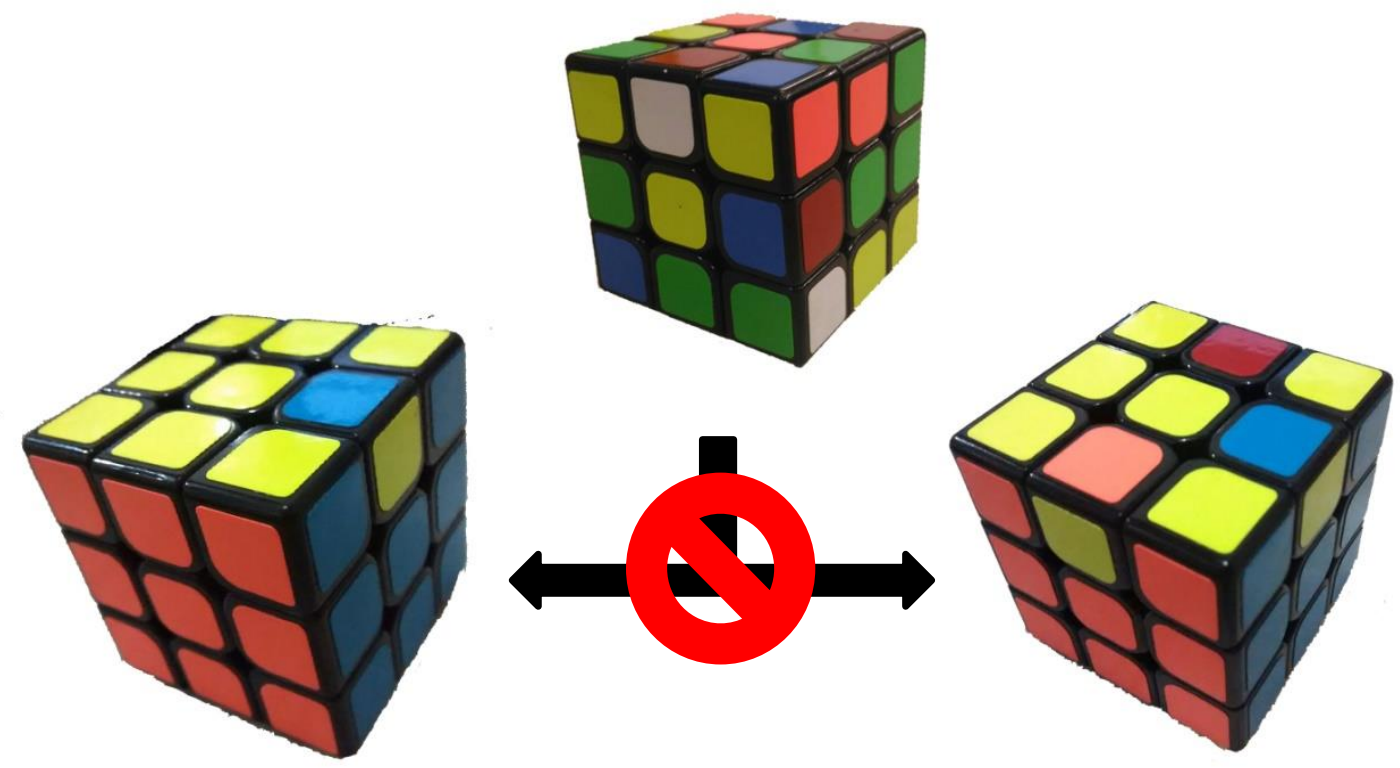

Figura 8-Caso impossível dos meios

Assim dividindo $\left(\frac{2^{12}}{2^{2}}\right)$, teremos $2^{10}$ permutações. Após estudar cada um dos casos apresentados, unificaremos todas as permutações.

Portanto, o produto de todas as permutações possíveis $8 ! \cdot 12 ! \cdot 3^{7} \cdot 2^{10}=$ 43.252.003.274.489.856.000 ou 43 quintilhões.

\section{DISCUSSÃO}

Para o estudo das combinações da montagem do cubo mágico foi necessário um conhecimento abrangente de permutações, arranjos combinatórios, permutações simples e com repetições, todo este estudo foi efetuado através de resolução de exercícios e seminários apresentados durante o desenvolvimento do trabalho, possibilitando verificar atuação da matemática em um simples objeto. Deste modo foram verificados especificamente cada estrutura do cubo, perante seus movimentos, métodos combinatórios de possíveis e impossíveis casos.

\section{CONCLUSÃO}

Através do presente trabalho foi possível ver a dinâmica de um quebra cabeça tridimensional e sua complexidade. A partir de cálculos combinatórios, analisando especificamente cada parte do cubo podendo identificar todas suas possibilidades de montagem, a partir de um simples quebra cabeça, para seu número finito inimaginário de possibilidades. 0 grande invento de Erno Rubiks tomou décadas, anos se passaram, adultos e crianças continuam tentando montar o quebra cabeça, incentivando e explorando o raciocínio lógico, como dizia Erno Rubiks, "Se você está curioso, você vai encontrar os quebra-cabeças em torno de você. Se você está determinado, você vai resolvê-los".

Foi possível concluir após o estudo de todas as possíveis soluções combinatórias do Cubo Mágico, que com a exposição de práticas desperta no aluno a curiosidade e motivação para o aprendizado de conceitos matemáticos. 


\section{REFERÊNCIAS}

CARVALHO, P. C. P. Métodos de Contagem e Probabilidade. Rio de Janeiro: IMPA, 2013.

CINOTO, R. Análise Combinatória. Disponível em: < http://www.cinoto.com.br/website/index. php/ancomb > Acesso em 21 de julho de 2016.

FARJADO, V. Cubo Mágico ajuda a aprender mais sobre a Matemática. Disponível em: <http://g1.globo.com/educacao/noticia/2013/01/cubo-magico-ajuda-aprender-mais-sobrematematica-diz-colecionador.html> Acesso em 20 de julho de 2016.

FOMIN, D; GENKIN, S; ITENBERG, I. Círculos Matemáticos, Tradução de Valéria de Magalhães lório. Rio de Janeiro: IMPA, 2012.

RUBIK'S, The Home of Rubik's Cube. The History of the Rubik's Cube. Disponivel em: <http://www.rubiks.com/World/Rubiks\%20history.aspx> Acesso em 20 de julho de 2016. 